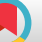

\title{
Public Adults Information Seeking Behaviors During Early Weeks of COVID-19 Epidemic: Early Lessons for Improvement of Epidemic Communication
}

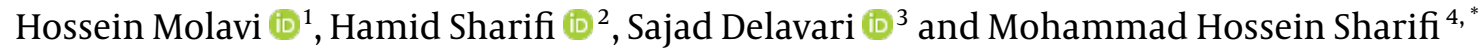 \\ ${ }^{1}$ Health Policy Research Center, Institute of Health, Shiraz University of Medical Sciences, Shiraz, Iran \\ ${ }^{2}$ HIV/STI Surveillance Research Center, and WHO Collaborating Center for HIV Surveillance, Institute for Futures Studies in Health, Kerman University of Medical Sciences, \\ Kerman, Iran \\ ${ }^{3}$ Health Human Resources Research Center, School of Management and Information Sciences, Shiraz University of Medical Sciences, Shiraz, Iran \\ ${ }^{4}$ Research Center for Traditional Medicine and History of Medicine, Shiraz University of Medical Sciences, Shiraz, Iran \\ "Corresponding author: Research Center for Traditional Medicine and History of Medicine, Shiraz University of Medical Sciences, Shiraz, Iran. Email: \\ mhsharifi1350@gmail.com
}

Received 2020 July 13; Revised 2020 September 21; Accepted 2020 October 09

\begin{abstract}
Background: Public support plays a crucial in managing public health crises. Communicating with the public during a pandemic has a major role in gaining public support. Public information-seeking behaviors are the core element of epidemic communication.. Objectives: The current study aimed to investigate the Iranian information-seeking behaviors during the first three weeks of the COVID-19 pandemic in Iran.

Methods: In this cross-sectional study, the use of web search queries to monitor the COVID-19 pandemic in Iran from December 2019 to March 12020 , is investigated. The Iranian search queries for COVID-19 were evaluated from December 30, 2019, to March 1, 2020. Google trend reports were used to retrieve data on the number of search queries. Queries were categorized into "epidemic news", "necessary protective equipment", "prevention strategies", and "treatment”. To analyze the data, segmented regression was applied. Also, the daily percent change (DPI) was estimated.

Results: The frequency of Google searches for COVID19-related queries first increased during the period of 18 - 23 February 2020 (DPC: 34.0; P value < 0.001), and then declined to March 1, 2020 (DPS:-3.9; P value < 0.001). The most prevalent query was "Epidemic news" (54\%), followed by "necessary equipment" (33\%). The frequency of these two queries increased from 18 - 23 February. "Prevention strategies" was the most common search category on March 1.
\end{abstract}

Conclusions: When an epidemic begins to spread, people try to get the "latest news" and "what they need to protect themselves".

Keywords: Coronavirus, Covid-19, Information-Seeking Behavior, Communication Media, Epidemics

\section{Background}

Public support is an essential component of coping with public health emergencies, such as the COVID-19 pandemic (1). Appropriate communication with the public at emergencies plays a key role in promoting public trust in health organizations, which in turn increases public support (2). Being proactive, transparent, and timely are crucial components of an appropriate public communication strategy during a pandemic. Also, at various stages of an epidemic, people seek different information. Therefore providing information that are tailored to each stage by the most suitable routes is necessary.

Early monitoring of information-seeking behaviors will guide policy-makers towards more appropriate deci- sions and more effective strategies for epidemic public communication. Although plenty of traditional sources of information are available, the internet is a major source of information gathering worldwide. Therefore, analyzing internet searches is one of the easily available sources to investigate public information-seeking behaviors (i.e., frequency of internet search queries) (3).

Globally millions of searches occur through the internet every day. Google, which handles billions of searches every day, has developed the Google Trends that analyzes the popularity of search queries separated by geographical region and date (https://trends.google.com). Google Trend is a valid tool to predict epidemics with a lag of one to three weeks in regions with a high internet penetration rate (47). 
As people search for what they think, Google searches can be considered as an early warning or monitoring system for the epidemic. Besides, such information can be used to identify public behaviors, concerns, or needs $(7,8)$. Therefore, it can be used as an early warning tool to predict what the public will search for during the next week(s). At the beginning of the COVID-19, misleading information and rumors began to spread all around the world that have challenged health authorizes, which resulted in withholding information from the public in countries such as the USA, Iran, Turkey, UK. Inappropriate communication strategies, along with misleading information, may reduce public support, which makes it more difficult to cope with the pandemic. Therefore, in the case of the COVID-19 pandemic, early investigation of public information-seeking behaviors can provide evidence for developing more appropriate communication strategies.

\section{Objectives}

According to the World Bank data, the internet penetration rate has been on the rise during the past decades in Iran, and more than 49 million Iranians (60\%) have used the internet in 2017(9). Accordingly, as a case study, the current study aimed to investigate the Iranian informationseeking behaviors during the first three weeks of the COVID-19 pandemic by analyzing data available on the Google Trends database.

\section{Methods}

In this cross-sectional study, the use of web search queries to monitor the COVID-19 pandemic in Iran from December 2019 to March 1, 2020, is investigated. When deciding about the study period, the date of pandemic beginning in China (December 30, 2019) and Iran (February 19, 2020) were considered. As people are more likely to search in their native languages, we also considered terms such as "CORONA" in the Persian language.

We retrieved all available data from Google Trends about the relative frequency of what Iranians searched for on the web. Search queries were categorized based on search topics. Search topics were categorized into four groups: 1) epidemic news; 2) equipment necessary for preventing the infection, such as mask and sanitizers; 3 ) latest prevention strategies; and 4) currently available treatments, such as symptoms and diagnosis methods.

The relative frequency of each search topic was calculated by summing up the relative frequency of all search queries categorized in a topic. The overall relative frequency of each topic was compared with the relative frequency of almost all COVID19-related searches in a defined period. Segmented regression was used to identify probable join-points and confidence intervals (CI). Daily percent change ((frequency of today's search queries divided by the frequency of yesterday's search queries)*100); DPC) and its 95\% CI were estimated for trends of the relative frequency of each topic. Data were analyzed using MS Excel and joinpoint regression software (Release 4.7.0).

\section{Results}

Trend of COVID19-related search queries was first increased from 18 to 23 February 2020 (DPC: 34.0; P-value < 0.001) and then declined from 23 February to 1 March 2020 (DPC: -3.9; P-value < 0.001). During the first week of the epidemic, "Epidemic news" was the most common search topic (54\% of all Google searches at its highest frequency), followed by the "necessary equipment for self-protection" (33\%) (Figure 1).

The "available prevention strategies" was the most common search topic on March 1, 2020. The trend of searches on this topic was increasing from February 18 to March 1 (DPC: 1.0; P-value < 0.001). Trend of searches for the "epidemic news" was first increased from 18 to $23 \mathrm{Feb}$ (DPC:16.6; P-value < 0.001) and then declined from 23 to 28 February(DPC:-10.2; P-value $<0.001$ ). The trend of searches for the "necessary equipment for self-protection" was first increasing from 19 to February 23 (P-value $<0.001$ ) and then declined from 23 to February 27 (P-value $<0.001$ ). The trend of searches for the "treatment-related" queries was increasing from 19 to February 23 (DPC: 0.2; P-value < 0.001; Figure 1).

\section{Discussion}

Based on the findings, "Epidemic News" and "necessary equipment for self-protection" were the most widely used keywords during the first three weeks after the beginning of the pandemic in Iran. Also, the trend of COVID-19-related search queries first increased from February 18, 2020, and then declined from February 23, 2020. The trend of search queries on the "available prevention strategies" had a rising trend since the onset of the pandemic.

During the first days of the pandemic expansion in Iran, "epidemic news" was the most widely used query. Probably mistrust to state TV news has contributed to this issue. This mistrust was amplified by rumors propagated by some foreign news agencies, and also local news agencies were claiming that Iran officials tried to cover up the pandemic expansion (10). Hence, people were trying to obtain information from other sources. It's widely believed that the scientific community is the most independent 


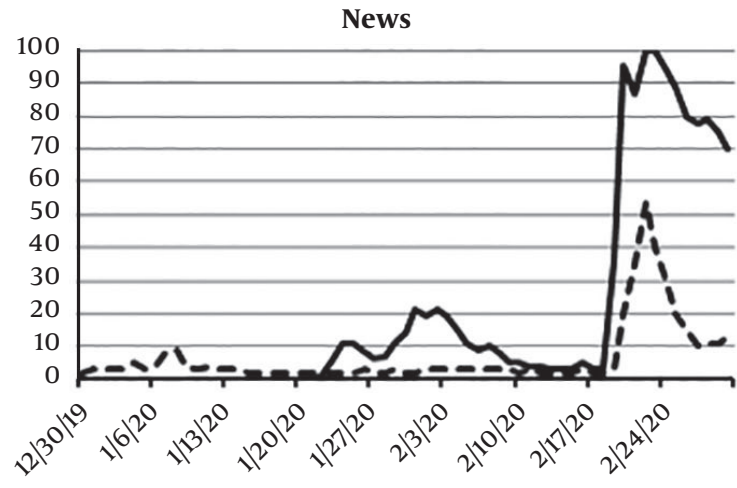

Prevention

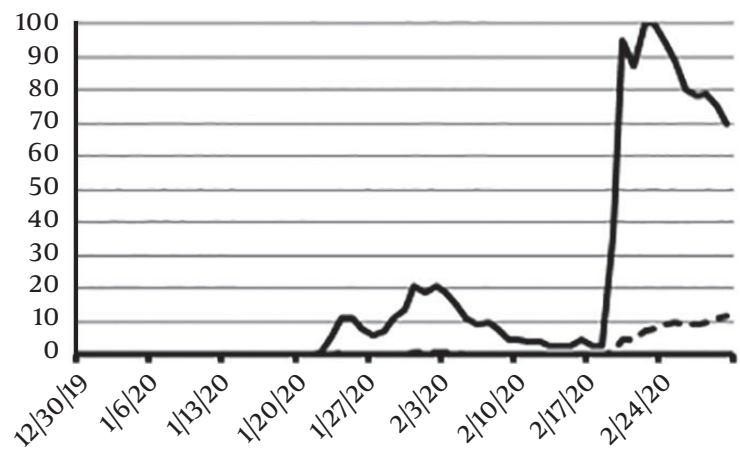

Lack of Equipment

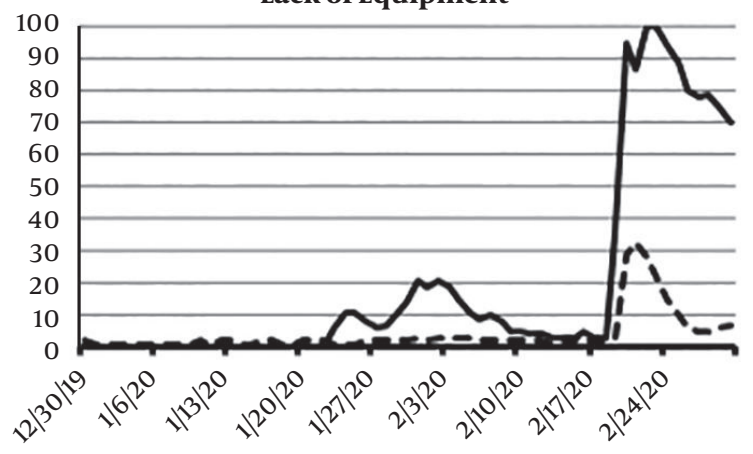

Symptoms \& Diagnosis

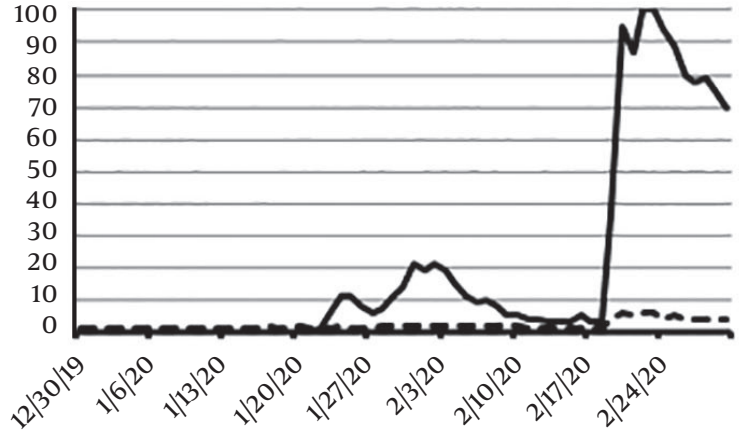

*Solid Lines: Relative Frequency of all COVID-related Queries Searched by Iranians m Google; Dashkd Lines: Topic Specific Relative Frequency

Figure 1. Relative frequency of Google searches about the COVID-19 epidemic by Iranians separated search topics.

source of information (11). Therefore, the on-time publication of scientific information by well-known academic persons helps to reduce panic $(12,13)$.

The first cases of COVID-19 in Iran were announced on February 19, 2020, by the Ministry of Health and Medical Education (MoHME). By considering a lag of seven days for predicting the pandemic expansion in Iran by Google searches, the first cases of the disease should have been identified on February 18, 2020 (4-7). However, some authors have argued that Google Trends predicts the epidemic with a lag of three weeks, assuming this scenario, the starting date of the epidemic in Iran, maybe February 4, 2020 (4-7), which seems more rational. Therefore, in the absence of early warning systems, the MoHME has announced the epidemic with an acceptable lag. However, considering the fast rotation of news in social media and the possibility of propagating rumors by politicians and those with bad economic intentions, this communication strategy seems irrational. The MoHME could enhance public trust by communicating with society and informing the people about the beginning of the COVID-19 epidemic. Further studies are needed to investigate what would have been occurred if the aforementioned communication strategy was used.

During the first days of the epidemic, people were mostly focused on the epidemic news, rather than how to protect themselves or their loved ones, because of public panic amplified by rumors. Therefore, even if appropriate content about preventive strategies was available on the web, they wouldn't trust them during the first days of the epidemic in Iran. Public behavior during the first days of an epidemic has major effects on the shape of the epidemic curve. Previous studies have indicated that coordinated messaging in public information campaigns is necessary to manage epidemics, providing reliable information to the public and various stakeholders, and countering misinformation and rumors (14). Besides, to appropriately respond to public health emergencies, the dissemination of 
news should be adjusted.

Moreover, the health literacy of society concerning how to use social media during an emergency is necessary. The second most widely used query during the first days of the epidemic was "necessary equipment for selfprotection". Therefore, in a proactive and on-time strategy for public epidemic communication, information about "where necessary equipment can be find?" should be available for all people before the epidemic onset (15). In this line, health authorities should not only estimate the necessary equipment required in different phases of the epidemic before its onset but also should design the most appropriate ways to distribute this equipment. Afterward, they should inform the public about "what they will need to protect themselves?" and "where they can find these equipments?", before the epidemic onset. Developing an information campaign is one of the most appreciable methods for rapid and reliable dissemination of information in society, particularly for equipment that are scarce (16). However, more advanced early warning systems are needed to enable health systems to perform epidemics risk assessment and communication. Therefore, the global community should move towards designing and implementing programs to enable developing counties to implement such early warning systems.

Using Google Trends to analyze information-seeking behavior is the main strength of this study, regarding that Google is the most widely used search engine. But the study had limitations also, including not investigating information-seeking behaviors in popular social media such as WhatsApp, Instagram, and Telegram, due to the unavailability of trends data.

In conclusion, although the MoHME has not concealed the epidemic information, it has not used an appropriate strategy to communicate with the public both before and during the COVID-19 epidemic. Iranian health policy-makers should have been prepared for the epidemic. Therefore, the necessary equipment should have been prepared. On the other hand, they were supposed to predict behaviors of the public during different phases of the epidemic, and to develop communication strategies (for both before the onset of / and during the epidemic). When an epidemic begins, people try to gather the "epidemic news" and "how to protect themselves", therefore appropriate communication strategies have to support the public in this regard.

\section{Footnotes}

Authors' Contribution: All authors acknowledge have participated in the work in a substantive way and are pre- pared to take full responsibility for the data presented herein according to the authorship criteria.

Conflict of Interests: The authors declare no potential conflict of interest with respect to the research, authorship, and publication of this article.

Funding/Support: There is no funding support.

\section{References}

1. Światecka A, Debiński W. Planning actions in the event of an epidemic of infectious diseases. Pol J Med, Bioeng Psychol. 2018;23(2):28-36. doi: 10.13174/pjambp.16.07.2018.04.

2. WHO. World Health Organization Outbreak Communica tion Planning Guide. Geneva: World Health Organization; 2008. Available from: https://www.who.int/ihr/elibrary/ WHOOutbreakCommsPlanngGuide.pdf.

3. Choi H, Varian HAL. Predicting the present with google trends. Econ Rec. 2012;88:2-9. doi: 10.1111/j.1475-4932.2012.00809.x.

4. Kang M, Zhong H, He J, Rutherford S, Yang F. Using Google Trends for influenza surveillance in South China. PLoS One. 2013;8(1). e55205. doi: 10.1371/journal.pone.0055205. [PubMed: 23372837]. [PubMed Central: PMC3555864].

5. Olson DR, Konty KJ, Paladini M, Viboud C, Simonsen L. Reassessing Google Flu Trends data for detection of seasonal and pandemic influenza: a comparative epidemiological study at three geographic scales. PLoS Comput Biol. 2013;9(10). e1003256. doi: 10.1371/journal.pcbi.1003256. [PubMed: 24146603]. [PubMed Central: PMC3798275].

6. Dugas AF, Hsieh YH, Levin SR, Pines JM, Mareiniss DP, Mohareb A, et al. Google Flu Trends: correlation with emergency department influenza rates and crowding metrics. Clin Infect Dis. 2012;54(4):4639. doi: 10.1093/cid/cir883. [PubMed: 22230244]. [PubMed Central: PMC3404718].

7. Cho S, Sohn CH, Jo MW, Shin SY, Lee JH, Ryoo SM, et al. Correlation between national influenza surveillance data and google trends in South Korea. PLoS One. 2013;8(12). e81422. doi: 10.1371/journal.pone.0081422. [PubMed: 24339927]. [PubMed Central: PMC3855287].

8. Polgreen PM, Chen Y, Pennock DM, Nelson FD. Using internet searches for influenza surveillance. Clin Infect Dis. 2008;47(11):1443-8. doi: 10.1086/593098. [PubMed: 18954267].

9. Bank W. International telecommunication union, world telecommunication/ICT development report and database, and world bank estimates. ITU; 2019. Available from: https://www.itu.int/en/ITU-D/Statistics/ Pages/publications/wtid.aspx.

10. Alam F, Shaar S, Nikolov A, Mubarak H, Martino GDS, Abdelali A, et al. Fighting the COVID-19 Infodemic: Modeling the Perspective of Journalists, Fact-Checkers, Social Media Platforms, Policy Makers, and the Society. arXiv preprint arXiv:2005.00033. 2020.

11. Academia Research. What sources can be considered as credible? 2014 Available from: https://www.academia-research.com/freelancewriting/crediblenon-credible-sources/.

12. Song P, Karako T. COVID-19: Real-time dissemination of scientific information to fight a public health emergency of international concern. Biosci Trends. 2020;14(1):1-2. doi: 10.5582/bst.2020.01056. [PubMed: 32092748].

13. Heymann DL, Shindo N. COVID-19: what is next for public health? Lancet. 2020;395(10224):542-5. doi:10.1016/s0140-6736(20)30374-3.

14. Lin L, Savoia E, Agboola F, Viswanath K. What have we learned about communication inequalities during the H1N1 pandemic: a systematic review of the literature. BMC Public Health. 2014;14(1). doi: 10.1186/14712458-14-484. 
15. Denecke K, Atique S. Social media and health crisis communication during epidemics. Participatory health through social media. Amsterdam, Netherland: Elsevier; 2016. p. 42-66. doi: 10.1016/b978-0-12809269-9.00004-9.
16. Tzeng HM. Nurses' professional care obligation and their attitudes towards SARS infection control measures in Taiwan during and after the 2003 epidemic. Nurs Ethics. 2004;11(3):277-89. doi: 10.1191/096733004ne6950a. [PubMed: 15176641]. 